\title{
Successful Treatment of Severe Hyperammonaemia with Ultra-High Dose Continuous Veno-Venous Haemodiafiltration
}

\author{
Dirk P. Boer ${ }^{a}$ Sarah L. Mourik ${ }^{b}$ Martijn W.F. van den Hoogen ${ }^{c}$ \\ Janneke G. Langendonk ${ }^{d}$ Hilde R.H. de Geus ${ }^{a}$ \\ ${ }^{a}$ Department of Intensive Care, Erasmus Medical Centre, Rotterdam, The Netherlands; ${ }^{b}$ Department of Obstetrics \\ and Gynaecology, Erasmus MC, Rotterdam, The Netherlands; ' Department of Nephrology, Erasmus MC, Rotterdam, \\ The Netherlands; ${ }^{d}$ Department of Internal Medicine-Metabolic Diseases, Rotterdam, The Netherlands
}

\section{Keywords}

Hyperammonaemia · Continuous veno-venous

haemodiafiltration · Renal replacement therapy

\section{Abstract}

Hyperammonaemia is a severe condition and often requires a multimodal treatment regimen. Dialysis has been described as a potential treatment option, but currently it is not the standard of care. In this report, we describe a case of a 40-year-old postpartum woman who developed severe hyperammonaemia due to liver failure and acute kidney injury (AKI) combined with a large intra-abdominal haematoma producing nitrogen waste products. She was treated successfully with continuous veno-venous haemodiafiltration using an ultra-high effluent rate $(100 \mathrm{~mL} / \mathrm{kg} / \mathrm{h})$ and was discharged alive 32 days after the initial admission. Our report indicates that successful ammonia clearance in the setting of AKI can be obtained only by using this high effluent rate. This treatment modality should be considered in all patients with $\mathrm{AKI}$ and severe hyperammonaemia when other treatment modalities fail to lower ammonia levels within hours to prevent irreversible but preventable neurological damage.

(c) 2019 The Author(s)

Published by S. Karger AG, Basel

\section{Introduction}

Severe hyperammonaemia, especially in the absence of structural liver disease, is a rare, life-threatening condition. Treatment consists of reducing ammonia production by treating underlying causes and increasing ammonia excretion. Successful extracorporeal ammonia clearance is an often-described option, but this option is not yet the standard of care when other treatment options fail [1-3].

\section{Case Report}

This case report describes a 40-year-old woman who developed a postpartum coma due to severe hyperammonaemia. She had a medical history of 2 uneventful vaginal births. In this pregnancy, labour was induced at 37 weeks of gestation. The indication was intermittent vaginal blood loss of an unknown cause. At $4 \mathrm{~cm}$ dilation, placental abruption was suspected, and an emergency caesarean section was performed. This case was complicated by postpartum haemorrhage (blood loss, 1,500 mL) for which sulprostone, a synthetic prostaglandin E2 derivative, was given. While in recovery, she developed seizures, for which magnesium sulfate was given, and she was admitted to the intensive care unit (ICU). Several hours postpartum, disseminated intravascular coagulation occurred, and a

\section{KARGER}

E-Mail karger@karger.com www.karger.com/bpu
Dirk P. Boer

Department of Intensive Care

Erasmus Medical Centre, Postbus 2040

NL-3000 CA Rotterdam (The Netherlands)

E-Mail dpboer@gmail.com 
Fig. 1. Serum ammonia level during admission to our hospital. The chequered line is treatment with CVVHD. The straight line is treatment with ultra-high effluent CVVHDF. The dashed line is treatment with half the dose of CVVHDF.

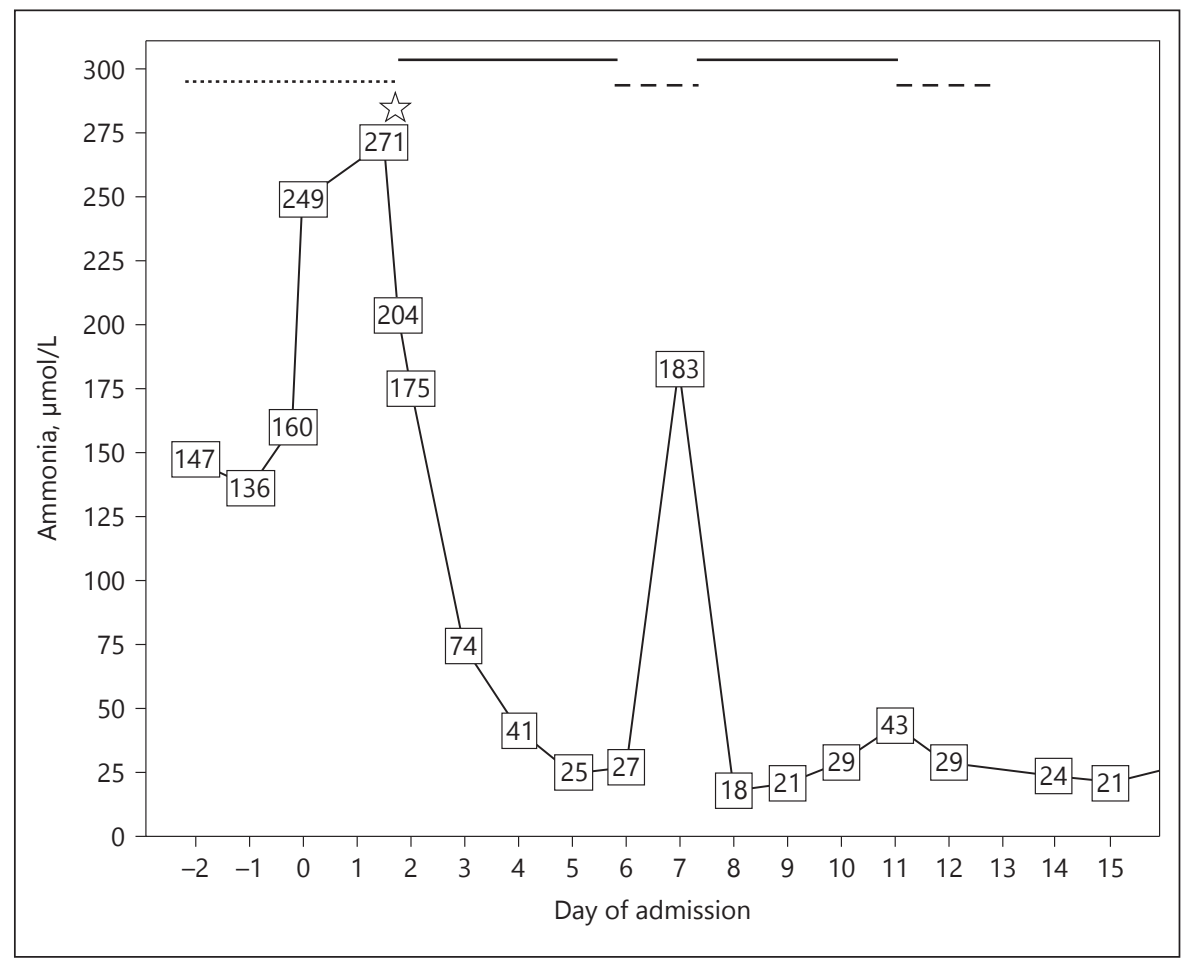

second laparotomy was necessary due to signs of active intra-abdominal bleeding. An arterial parametrial bleed was stopped after embolization, and haemodynamic stability was obtained. Total blood loss was $5 \mathrm{~L}$, for which multiple transfusions were necessary.

Her ICU stay was complicated by the presence of acute kidney injury (AKI) and ischaemic liver injury, both after low perfusion due to hypovolemia. Anuria developed, and continuous veno-venous haemodialysis (CVVHD) was initiated. Hyperammonaemia (147 $\mu \mathrm{mol} / \mathrm{L}$ quickly rising to $249 \mu \mathrm{mol} / \mathrm{L}$ ) with cerebral oedema, as shown on a CT scan, and subsequent reoccurrence of seizures started 3 days after the initial surgical intervention. Intracranial pressure (ICP) monitoring was initiated, for which she was transferred to our hospital on the fifth day after the initial admission. This day is denoted as day 0 in Figure 1. ICP monitoring revealed low ICP $(10 \mathrm{~mm} \mathrm{Hg})$.

The hyperammonaemia was first considered to be related to liver failure with massive amounts of nitrogen produced from both the pelvic haematoma and uterine necrosis following earlier arterial embolization. Therefore, rifaximin and lactulose were given. However, due to the minor response to this treatment and the discrepancy between the level of ammonia $(249 \mu \mathrm{mol} / \mathrm{L})$ and the other liver enzyme and function tests, which were almost normal, a primary inherited urea acid cycle defect was suspected. This patient tested negative based on extensive biochemical and DNA testing. Treatment was intensified with carbohydrate infusion, sodium benzoate, arginine and carnitine without a sufficient effect; ammonia levels decreased to only $200 \mu \mathrm{mol} / \mathrm{L}$, and there was no clinical improvement. Renal replacement therapy with CVVHD was already ongoing. On the second day of admission to our hospital (day 2 in Fig. 1), one session of intermittent haemodialysis was started. Settings for the intermittent haemodialysis were a blood flow of $400 \mathrm{~mL} / \mathrm{min}$ and dialysate flow of $500 \mathrm{~mL} / \mathrm{min}$ for $4 \mathrm{~h}$. The session of intermittent haemodialysis was followed by continuous veno-venous haemodiafiltration (CVVHDF) with 2 CRRT machines resulting in an ultra-high effluent rate $(100 \mathrm{~mL} /$ $\mathrm{kg} / \mathrm{h})$. The patient's estimated body weight was $85 \mathrm{~kg}$. For each of the CRRT machines, the settings were as follows: blood flow, 160 $\mathrm{mL} / \mathrm{min}$; substitution flow, $1,400 \mathrm{~mL} / \mathrm{h}$; and dialysate flow, 2,800 $\mathrm{mL} / \mathrm{h}$. We used the Ultraflux polysulfan AV1000S filter with multiFiltratePRO (Fresenius Medical Care, Germany). Anticoagulation was sought with heparin, aiming for an APTT of 50-70 s.

The ammonia levels subsequently decreased to $<100 \mu \mathrm{mol} / \mathrm{L}$ within $24 \mathrm{~h}$, and the patient regained consciousness for the first time. Figure 1 shows the trend of the serum ammonia level in our patient starting 2 days before transfer to our hospital. Day 0 was the day of admission to our hospital. During her stay in our hospital, the patient was haemodynamically stable without the need of vasopressors. After regaining consciousness, she was able to be extubated without any problems.

After 5 days of high-flow CVVHDF (day 6 in Fig. 1), the effluent rate was reduced to a single machine, which resulted in a rebound of the ammonia level and decreased consciousness. Reinitiating ultra-high haemodiafiltration reduced ammonia levels rapidly. Although the treatment for hyperammonaemia was multimodal, this rebound caused by the reduction in the dialysis dose suggests that CRRT was the necessary additive therapy to reduce plasma ammonia levels.

On the 10th day of admission to our hospital, the patient's liver function was further improved, nitrogen waste products were reduced, and ultra-high haemodiafiltration was able to be stopped. Two weeks after admission, our patient was discharged from the ICU, and 32 days after childbirth, she was discharged home. Her renal and liver function tests normalized, and physical and mental recovery was reported. However, it was observed that mild cognitive dysfunction persisted at the first outpatient clinic visit. 


\section{Discussion/Conclusion}

The clearance of ammonia can be obtained using dialysis, either with intermittent haemodialysis or with continuous methods. A drawback of the continuous method is the relatively slow clearance compared to intermittent haemodialysis. To provide sufficient clearance, multiple intermittent sessions are often given, but these sessions are labour-intensive and do not tackle the problem of rebound hyperammonaemia. Therefore, an ultra-high dose of CVVHDF can provide the best of both worlds: high clearance without the risk of rebound hyperammonaemia. Nonetheless, 2 CVVHDF machines require 2 separate venous access sites with the associated risks of bleeding and infection. The reason for using 2 separate machines was that the multiFiltratePRO allows efficient dialysis to a maximum of $4,000 \mathrm{~mL} / \mathrm{h}$ in effluent flow rate. Beyond 4,000 $\mathrm{mL} / \mathrm{h}$, dialysis efficiency decreases due to saturation of the dialysate half of the membrane. In the other half of the membrane, there will be back diffusion into the plasma compartment. The increase in blood flow is not sufficient to compensate for this phenomenon. Thus, effluent flow is the compromising factor for clearance, not the blood flow. Therefore, if we would have increased effluent volume, this would not have efficiently increased ammonia clearance, since we would have created back flow. Local logistics did not allow us to treat our patient in a semi-continuous mode (i.e., SLED modality).

Nevertheless, this treatment is not the standard of care. This situation could first be due to the potential risks (i.e., placement of a central venous catheter, infection risk, and the use of anticoagulation) and second, for its costs. In addition, as far as we know, very little evidence has been published for treating hyperammonaemia by renal replacement therapy. In addition to these reasons, the rarity of this condition, in combination with a lack of expertise and knowledge, can also explain why this treatment is not performed often. However, we demonstrated in this case report that when other treatment modalities fail to lower ammonia levels in patients with raised ICP, extracorporeal re- moval with an ultra-high filtration rate should be considered to prevent neurological damage. Especially in patients with concurrent diseases and hyperammonaemia, quick and adequate clearance should be pursued because of the potential risks in very high and quickly rising levels of ammonia.

Our report shows that CVVHDF can successfully clear ammonia. In the presented case, ammonia clearance was best obtained by using an ultra-high effluent rate. This outcome confirms that while AKI persists, a high effluent rate is necessary to clear the high ammonia levels.

In conclusion, we state that a high effluent rate of CVVHDF is a sufficient treatment modality for removal of ammonia in selected cases of patients with hyperammonaemia (i.e., in the setting of AKI when other treatment modalities fail) to prevent severe neurological damage. The exact effluent rate and the duration of the therapy should be patient-specific.

\section{Statement of Ethics}

The authors have no ethical conflicts to disclose. The authors obtained informed consent from the subject of our case report to publish this case.

\section{Disclosure Statement}

The authors declare that they have no conflicts of interest to disclose.

\section{Funding Sources}

No funding was received.

\section{Author Contribution}

D.P.B. is the first author of this case report and wrote the manuscript. Supervision and correction were done by H.R.H.G. S.L.M, M.W.F.H., and J.G.L. contributed by correcting the manuscript by focusing on the details in obstetrics, nephrology and metabolic conditions respectively.

\section{References}

1 Gupta S, Fenves AZ, Hootkins R. The role of RRT in hyperammonemic patients. Clin J Am Soc Nephrol. 2016 Oct;11(10):1872-8.

2 Cardoso FS, Gottfried M, Tujios S, Olson JC, Karvellas CJ; US Acute Liver Failure

Treatment of Hyperammonaemia by CVVHDF
Study Group. Continuous renal replacement therapy is associated with reduced serum ammonia levels and mortality in acute liver failure. Hepatology. 2017 Aug;67(2): 711-20.
3 Slack AJ, Auzinger G, Willars C, Dew $\mathrm{T}$, Musto R, Corsilli D, et al. Ammonia clearance with haemofiltration in adults with liver disease. Liver Int. 2014 Jan;34(1): $42-8$. 